Physics

Physics Research Publications

Correlation energy in a spin-polarized two-dimensional electron liquid in the high-density limit
S. Chesi
G. F. Giuliani

This paper is posted at Purdue e-Pubs.

http://docs.lib.purdue.edu/physics_articles/741 


\title{
Correlation energy in a spin-polarized two-dimensional electron liquid in the high-density limit
}

\author{
Stefano Chesi and Gabriele F. Giuliani \\ Department of Physics, Purdue University, West Lafayette, Indiana 47907, USA
}

(Received 21 November 2006; published 18 April 2007)

\begin{abstract}
We have obtained an analytic expression for the ring diagram contribution to the correlation energy of a two-dimensional electron liquid as a function of the uniform fractional spin polarization. Our results can be used to improve the interpolation formulas which represent the basic ingredient for the construction of modern spin-density functionals in two dimensions.
\end{abstract}

DOI: 10.1103/PhysRevB.75.153306

PACS number(s): 71.10.Ca, 71.15.Mb, 71.45.Gm, 72.25.Dc

The electron liquid, an ideal model in which electrons interact via a Coulomb potential in the presence of a uniform rigid neutralizing background, represents a fundamental paradigm for the understanding of condensed-matter systems. ${ }^{1}$ Although for homogeneous states the ground-state properties are completely determined by the number density $n$, it can be useful and often necessary to study the properties of the system in the presence of a given uniform spin polarization. Accordingly, for the case under consideration of a two-dimensional system, alongside the traditionally employed dimensionless density parameter $r_{s}=1 / \sqrt{\pi n a_{B}^{2}}$ we will consider the effects of polarization as parametrized by $p=\frac{2 S_{z}}{\hbar n}$, where $S_{z}$ is the uniform spin-polarization density.

While in the general uniform case the total energy of the system can be accurately obtained only by numerical means and is available in the form of interpolation formulas of Monte Carlo results, ${ }^{1,2}$ an exact analytic treatment is possible in the high-density limit in the form of a perturbative expansion in $r_{s}$, which in this case is vanishingly small. ${ }^{3}$ In this limit, the first-order correction to the noninteracting energy simply coincides with the familiar exchange energy, which has a simple analytic formula proportional to $r_{s}^{-1}$. The remaining correction is referred to as the correlation energy. ${ }^{1}$

The high-density expansion of the correlation energy was studied in the classic Ref. 4. The final expression for the paramagnetic case (in Rydberg units) reads

$$
\mathcal{E}_{c}\left(r_{s}, p=0\right)=-0.385-\frac{2 \sqrt{2}}{3 \pi}(10-3 \pi) r_{s} \ln r_{s}+\cdots,
$$

while the $p=1$ result can also be obtained by making use of a simple transformation. ${ }^{4}$

For generic values of the polarization, the coefficients of expansion (1) become functions of $p$.

The constant term in Eq. (1) is obtained from secondorder perturbation theory and is the sum of two distinct contributions. The first is the second-order exchange energy, which is independent of $p$ and can be calculated analytically. ${ }^{5}$ The second one stems from the second-order direct energy term, commonly referred to as the first ring diagram. The latter has been recently evaluated numerically for generic values of $p$ in Ref. 6. These contributions are accurately represented by the interpolation formula of Ref. 2 .

The next perturbative terms are in general divergent and a finite result is obtained upon exact summation of the infinite series of the most diverging contributions, the remaining ring diagrams. This leads to the subleading $r_{s} \ln r_{s}$ term.

This elegant method was originally developed for the corresponding unpolarized three-dimensional case, ${ }^{7}$ a problem in which the ring diagrams sum up to give the leading $\ln r_{s}$ contribution to the correlation energy. ${ }^{8}$ For the threedimensional case, the exact polarization dependence was also determined. ${ }^{9}$

The generic dependence of the $r_{s} \ln r_{s}$ term on $p$ is obtained in this work. The complete formula for the presently known leading terms of $\mathcal{E}_{c}\left(r_{s}, p\right)$ is provided for reference in the Appendix.

Following Ref. 4, the value of the generic diverging ring diagram of order $n$ is obtained from the expression

$$
f_{R}^{(n)}(p)=-\frac{(-1)^{n}}{\pi n r_{s}^{2}} \int_{-\infty}^{+\infty} d u \int_{0}^{\infty} q^{2} d q\left(\frac{Q_{q}(u) r_{s}}{2 \sqrt{2} \pi q}\right)^{n}
$$

where $n$ is a positive integer larger than 1 .

The explicit expression for $Q_{q}(u)$ is given by

$$
Q_{q}(u)=\sum_{\sigma=\uparrow, \downarrow} \int \frac{d \mathbf{k}}{q} \frac{q+2 k_{x}}{\left(\frac{q}{2}+k_{x}\right)^{2}+u^{2}} n_{\sigma}(k)\left[1-n_{\sigma}\left(k^{\prime}\right)\right],
$$

where $k^{\prime}=\sqrt{\left(k_{x}+q\right)^{2}+k_{y}^{2}}$. The polarization dependence of $Q_{q}(u)$ is implicitly determined by the occupation functions

$$
n_{\sigma}(k)=\theta\left(k_{\sigma}-k\right)
$$

For each of the two spin orientations, the Fermi wave vector is obtained from the relation

$$
k_{\uparrow(\downarrow)}=\sqrt{1 \pm p},
$$

where for convenience we have rescaled the wave vectors by $k_{F}=\sqrt{2 \pi n}$.

The expression corresponding to the generic ring diagram of Eq. (2) has a (formal) dependence of $r_{s}^{n-2}$, but in reality for $n \geqslant 3$ it involves a diverging integral. The sum to infinite order is 


$$
\begin{aligned}
f_{R}(p)= & \frac{1}{\pi r_{s}^{2}} \int_{-\infty}^{+\infty} d u \int_{0}^{\infty} q^{2} d q\left[\ln \left(1+\frac{Q_{q}(u) r_{s}}{2 \sqrt{2} \pi q}\right)-\frac{Q_{q}(u) r_{s}}{2 \sqrt{2} \pi q}\right. \\
& \left.+\frac{1}{2}\left(\frac{Q_{q}(u) r_{s}}{2 \sqrt{2} \pi q}\right)^{2}\right]
\end{aligned}
$$

which is instead a converging integral whose leading contribution in the $r_{s} \rightarrow 0$ limit is again proportional to $r_{s} \ln r_{s}$ and is determined by the small- $q$ integration region..$^{10}$ Accordingly, it is sufficient to employ here the limiting value $Q_{q=0}(u)$, as obtained from Eq. (3). The result can be written as

$$
Q_{0}(u)=2 \pi\left[R\left(u / k_{\uparrow}\right)+R\left(u / k_{\downarrow}\right)\right],
$$

where the function $R(u)$ is defined as

$$
R(u)=1-\frac{1}{\sqrt{1+1 / u^{2}}} .
$$

To this point, our discussion follows Ref. 4, where the implicit expression (6) as well as Eq. (7) were originally provided for a generic value of $p$. There, however, the explicit evaluation was only done for $p=0$. In the general case, using Eq. (7) and performing in Eq. (6) the wave-vector integration (up to an arbitrary upper limit), one extracts the leading contribution in $r_{s}$ :

$$
f_{R}(p) \simeq-\frac{r_{s} \ln r_{s}}{3 \sqrt{2}(2 \pi)^{4}} \int_{-\infty}^{+\infty}\left[Q_{0}(u)\right]^{3} d u,
$$

which gives the standard result (1) by making use of $Q_{0}(u)$ $=4 \pi R(u)$, i.e.,

$$
f_{R}(0) \simeq-\frac{2 \sqrt{2}}{3 \pi}(10-3 \pi) r_{s} \ln r_{s} .
$$

We find that the integral in Eq. (9) can be performed exactly in the general case. The result can be expressed in terms of the function

$$
F(x, y)=\int_{-\infty}^{+\infty}[R(u / x)]^{2} R(u / y) d u
$$

which has the explicit expression

$$
F(x, y)=4(x+y)-\pi x-4 x E\left(1-\frac{y^{2}}{x^{2}}\right)+\frac{2 x^{2} \arccos \frac{y}{x}}{\sqrt{x^{2}-y^{2}}} .
$$

Here, $E(x)$ is the complete elliptic integral of the second type, ${ }^{11}$ and one should use the identity $\frac{\arccos \frac{y}{x}}{\sqrt{x^{2}-y^{2}}}=\frac{\operatorname{arccosh} \frac{y}{x}}{\sqrt{y^{2}-x^{2}}}$ for $y>x$. At $p=0$, one only needs the value $F(1,1)=10-3 \pi$.

The result for the ring diagrams at finite polarization can be compactly and elegantly expressed in terms of the corresponding polarization scaling function $I_{R}(p)$, defined as

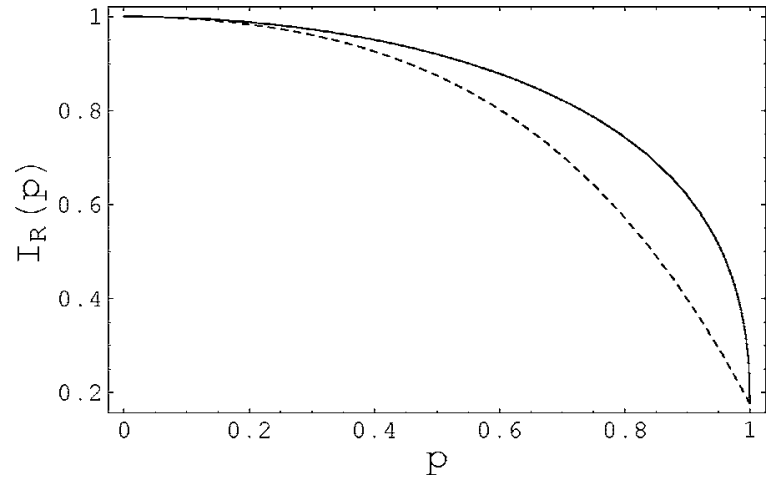

FIG. 1. Plot of the scaling factor $I_{R}(p)$ [defined in Eq. (13)] as a function of the fractional polarization $p$. Solid line: Exact expression (14). Dashed line: Eq. (15) as obtained from the interpolation formula proposed in Ref. 2.

$$
I_{R}(p)=\lim _{r_{s} \rightarrow 0} \frac{f_{R}(p)}{f_{R}(0)} .
$$

The final expression is given by

$$
I_{R}(p)=\frac{1}{8}\left(k_{\uparrow}+k_{\downarrow}+3 \frac{F\left(k_{\uparrow}, k_{\downarrow}\right)+F\left(k_{\downarrow}, k_{\uparrow}\right)}{10-3 \pi}\right),
$$

which readily gives the correct value at $p=0$. At $p=1$, using $F(\sqrt{2}, 0)=F(0, \sqrt{2})=0$, we obtain the known $\operatorname{result}^{4} I_{R}(1)$ $=\frac{\sqrt{2}}{8}$.

As it turns out, the exact result $I_{R}(p)$ is not well reflected in the most recent interpolation formulas of Monte Carlo calculations provided in the literature. In particular, from the correlation energy formula of Ref. 2, denoted here as $\mathcal{E}_{c}^{M C}\left(r_{s}, p\right)$, the following limit is obtained:

$$
\begin{aligned}
I_{R}^{M C}(p) & =\lim _{r_{s} \rightarrow 0} \frac{\mathcal{E}_{c}^{M C}\left(r_{s}, p\right)-\mathcal{E}_{c}^{M C}(0, p)}{f_{R}(0)} \\
& \simeq 1-0.3932 p^{2}-0.4297 p^{4},
\end{aligned}
$$

which is compared in Fig. 1 to the exact result of Eq. (14). The difference is remarkable, even if the specific aim of Ref. 2 is to address the polarization dependence of the whole correlation energy. Agreement of $I_{R}^{M C}(p)$ with the exact result is only achieved for $p=0$ and $p=1$, values known from the extant literature. A noticeable failure is the behavior near $p=1$, where the polynomial (15) gives a finite slope while the leading term in the exact expression is

$$
I_{R}(p) \simeq \frac{\sqrt{2}}{8}+\frac{14-3 \pi}{4(10-3 \pi)} \sqrt{1-p}
$$

Around $p=0$, while being correctly quadratic in $p$, Eq. (15) displays an incorrect coefficient. The exact coefficient is given by

$$
I_{R}(p) \simeq 1-\frac{168-45 \pi}{160(10-3 \pi)} p^{2} \simeq 1-0.2893 p^{2}
$$

The disagreement between $I(p)$ and the Monte Carlo based interpolation $I^{M C}(p)$ is hardly surprising, for the latter was obtained by sampling the energy at a number of polar- 
ization values for each of the values $r_{s}=1,2,5,10$, which are clearly outside of the $r_{s} \ll 1$ perturbative regime. ${ }^{12}$ In practice, $I_{R}(p)$ refers to the subleading term in the density expansion, so that it gives only small corrections to the total energy. Nevertheless, incorporating this exact formula would certainly result in an improved empirical expression for the polarization dependence of the correlation energy.

We conclude by noting that a similar, yet more involved, calculation can be carried out for the high-density limit of the electron liquid correlation energy in the presence of the Rashba spin orbit. An analysis of this interesting and timely problem is provided elsewhere. ${ }^{13}$

\section{APPENDIX}

For ease of reference, we collect here the explicit form of all the leading terms contributing to the perturbative expansion of the total energy of the two-dimensional electron liquid at finite polarization. The general formula (in Rydberg units) reads

$$
\mathcal{E}\left(r_{s}, p\right)=\mathcal{E}_{K}\left(r_{s}, p\right)+\mathcal{E}_{x}\left(r_{s}, p\right)+\mathcal{E}_{c}\left(r_{s}, p\right) .
$$

In this expression, $\mathcal{E}_{K}\left(r_{s}, p\right)$ represents the noninteracting kinetic energy and is given by

$$
\mathcal{E}_{K}\left(r_{s}, p\right)=\frac{1+p^{2}}{r_{s}^{2}}
$$

while $\mathcal{E}_{x}\left(r_{s}, p\right)$ represents the exchange energy,

$$
\mathcal{E}_{x}\left(r_{s}, p\right)=-\frac{8 \sqrt{2}}{3 \pi} \frac{(1+p)^{3 / 2}+(1-p)^{3 / 2}}{2 r_{s}} .
$$

The expansion for the correlation energy takes the form

$$
\mathcal{E}_{c}\left(r_{s}, p\right)=\mathcal{E}_{2}(p)-\frac{2 \sqrt{2}}{3 \pi}(10-3 \pi) I_{R}(p) r_{s} \ln r_{s}+\cdots
$$

where the omitted corrections are of order $\mathcal{O}\left(r_{s}\right)$. In this expression, $I_{R}(p)$ is defined by Eqs. (14), (12), and (5). The density independent term is in turn given by

$$
\mathcal{E}_{2}(p)=\mathcal{E}_{2}^{(b)}+\mathcal{E}_{2}^{(r)}(p),
$$

where ${ }^{5}$

$$
\mathcal{E}_{2}^{(b)}=0.2287
$$

and $^{6}$

$$
\mathcal{E}_{2}^{(r)}(p)=-0.6137 I_{2}(p)
$$

where the scaling function $I_{2}(p)$ is given by

$$
I_{2}(p)=1-\frac{(1+p) \ln (1+p)+(1-p) \ln (1-p)}{4 \ln 2}-\frac{\delta f(p)}{2},
$$

with $^{14}$

$$
\delta f(p) \simeq 0.0636 p^{2}-0.1024 p^{4}+0.0389 p^{6} .
$$

${ }^{1}$ G. F. Giuliani and G. Vignale, Quantum Theory of the Electron Liquid (Cambridge University Press, Cambridge, 2005).

${ }^{2}$ C. Attaccalite, S. Moroni, P. Gori-Giorgi, and G. B. Bachelet, Phys. Rev. Lett. 88, 256601 (2002).

${ }^{3}$ We assume throughout that the system is in a spatially homogeneous state. The possibility of spin or charge-density wave states as envisaged by Overhauser (Refs. 15-17) can be ruled out in the high-density regime as shown in G. F. Giuliani and G. Vignale (unpublished).

${ }^{4}$ A. K. Rajagopal and J. C. Kimball, Phys. Rev. B 15, 2819 (1977).

${ }^{5}$ A. Isihara and L. Ioriatti, Phys. Rev. B 22, 214 (1980).

${ }^{6}$ M. Seidl, Phys. Rev. B 70, 073101 (2004).

${ }^{7}$ M. Gell-Mann and K. A. Brueckner, Phys. Rev. 106, 364 (1957).

${ }^{8}$ At variance with the two-dimensional case, in three dimensions all the individual ring diagrams correspond to divergent contributions.
${ }^{9}$ Y. Wang and J. P. Perdew, Phys. Rev. B 43, 8911 (1991).

${ }^{10}$ Notice that by construction, the density independent contribution from the $n=2$ ring diagram has been omitted. This is the quantity studied in Ref. 6.

${ }^{11}$ M. Abramowitz and I. A. Stegun, Handbook of Mathematical Functions (Dover, New York, 1965).

${ }^{12}$ It should be kept in mind that in practice, the perturbative theoretic expression of the energy of the electron liquid based on the ring diagrams does provide a quantitavely accurate description only at very small values of $r_{s}$ (see also Ref. 1).

${ }^{13} \mathrm{~S}$. Chesi and G. F. Giuliani (unpublished).

${ }^{14}$ It should be noted that as in the original paper, Eq. (A9) does not account for a small dip near $p=1$ seemingly uncovered in the numerical work of Ref. 6.

${ }^{15}$ A. W. Overhauser, Phys. Rev. Lett. 4, 462 (1960).

${ }^{16}$ A. W. Overhauser, Phys. Rev. 128, 1437 (1962).

${ }^{17}$ A. W. Overhauser, Phys. Rev. 167, 691 (1968). 